\title{
RADIO SOURCE STRUCTURE AND UNIFIED MODELS
}

\author{
J. B. HUTCHINGS \\ Dominion Astrophysical Observatory, 5071 W. Saanich Rd \\ Victoria, B.C. V8X 4M6, Canada \\ and \\ S. G. NEFF \\ NASA Goddard Space Flight Center, Greenbelt \\ MD 20771, USA
}

\begin{abstract}
The results are presented of a program to study the radio structure of radio galaxies, and to compare them with quasars. The samples are matched in redshift and luminosity in the redshift range 0.2 to 1.0 , and mapped with the same VLA configuration. The quasar results have been published in earlier papers and the radio galaxy results are in press. We compare 100 quasars with 80 radio galaxies. The comparison shows the following radio galaxies differ from quasars in the following ways:

1) The overall sizes are larger for radio galaxies by 2 to 3 times.

2) There are few large bend angles in radio galaxies.

3) The lobe length ratio distribution differs.

4) The radio galaxies have no population of one-sided sources.

5) The lobe flux ratio has less range in the radio galaxies.

6) Radio galaxies have few core-dominated sources and many with no detectable core: few with comparable core and lobe flux.

7) The most luminous sources are lobe-dominated and more powerful than the quasars. The most luminous quasars are unresolved cores.

Points 1,2,3 can be fit with a single 'unified' model which has a distribution of sizes and bend angles up to $25^{\circ}$, with small expansion velocities. The quasars are viewed within a cone angle of $50^{\circ}$ and the radio galaxies outside the cone. However, the model does incorporate a source growth scenario and points 4 through 7 imply that there are instrinsic differences between the source types as well as orientation. We also discuss the limitations on beaming and bulk motions implied by the results.
\end{abstract}

Key words: radio galaxies, quasars, radio maps, unified models

T. J.-L. Courvoisier and A. Blecha: Multi-Wavelength Continuum Emission of AGN, 427.

C) 1994 IAU. Printed in the Netherlands. 